\title{
System Architecture in Cognitive Radio Networks using a Radio Environment Map
}

\author{
Anna Vizziello \\ Department of Electronics \\ Università degli Studi di Pavia \\ 27100, Pavia - Italy \\ anna.vizziello@unipv.it
}

\author{
Jordi Perez-Romero* \\ Department of Signal Theory and \\ Communications (TSC) \\ Universitat Politecnica de Catalunya (UPC) \\ 08034, Barcelona - Spain \\ jorperez@tsc.upc.edu
}

\begin{abstract}
Cognitive Radio (CR) is considered as a promising solution to improve wireless spectrum utilization. Moreover, through optimization frameworks, Radio Resource Management (RRM) procedures are designed to enhance the efficient utilization of resources in CR networks. To face this issue in a holistic perspective, the RRM solutions should take into account not only the optimization task but also their impact on the design task. Thus, in this paper, a system architecture of a novel RRM is proposed for CR networks. In particular, it is fundamental to construct flexible systems to work across heterogeneous systems and help realize their full potential. Therefore, in the proposed system, heterogeneous Primary Users (PUs) with multiple features and variable $\mathrm{CR}$ demands are considered. A Radio Environment Map (REM) is used to obtain the required PU features, which are exploited to improve the adaptability in CR networks and, thus, to design an efficient Cognitive RRM. The functionalities of the network elements are described in detail, along with the message exchange to carry out the resource management.
\end{abstract}

Index Terms - System Architecture, Radio Resource Management, Cognitive Radio Networks

\section{INTRODUCTION}

The wasteful static frequency assignment, fixed radio functionalities and architectures associated with current wireless systems have limited the achievable spectrum efficiency. Cognitive Radio (CR) is an intelligent device aiming at the efficient spectrum utilization and it can be exploited to overcome the limited spectrum efficiency of the classical wireless systems. Specifically, spectrum utilization can be significantly improved by allowing CR users to access the unused spectrum resources of the Primary Users (PUs). Additionally, CR has to vacate the spectrum band as soon as a PU

\footnotetext{
* The work of Dr. Jordi Perez-Romero was supported by the European Commission in the framework of the FP7 FARAMIR Project (Ref. ICT- 248351).
}

is detected in the same band to avoid interference towards PU.

The spectrum efficiency can be further enhanced through a Radio Resource Management (RRM) strategy. In fact, in wireless communication systems, RRM is the key control functionality that enables the efficient utilization of limited radio resources by developing optimization frameworks. As a result, combining a smart RRM optimization framework with the spectrum awareness and frequency agility of CR users, more efficient and flexible spectrum utilization can be achieved [1].

However, in existing Cognitive RRM frameworks, such as [3], [4], the authors are narrowly focused on the optimization task, and lack a holistic perspective on the problem [2]. To address this issue it is fundamental to consider the problems faced when designing RRM strategies and also how these RRM solutions impact on the design task of the system architecture [2].

Thus, in this work, after describing the scenario and problem statement of our Cognitive RRM solution [6], we detail the proposed system architecture, showing the network entities along with their functionalities.

Specifically, in [6] we exploit the opportunities provided by the heterogeneity of different PUs, also called PU types in the following. In fact, in existing Cognitive RRM systems, such as [3] and [7], all PUs' signals are observed as signals of higher access priority to spectral resources regardless of their type. Grouping these signals into a single abstract utilization category removes some PU information regarding specific features, such as PU allowable interference level, bandwidth and activity pattern, which can otherwise be exploited by the RRM system. The PU features have different values depending on heterogeneous PUs. In [6], the existence of a specific PU type influences the amount of available capacity for CR users. After calculating the available capacity of the CR network, the RRM regulates the sharing of available capacity among CR users. In particular, a cluster of CR users that share the same available resources is formulated around each PU type.

This paper presents the system architecture for the proposed Cognitive RRM [6], detailing the functionalities of the network elements and the messages exchange among them to carry out the resource management. Moreover, in this work, we use a Radio Environment Map (REM) to obtain the re- 
quired geo-localized spectral activities, policy information, and other RF environment information useful to estimate the available network resources. The REM, supported by $\mathrm{CR}$ network, is envisioned as the large scale navigator for CR users. It provides cognitive services to the associated internal networks as well as a useful awareness of external networks such as PU systems. REM covers multi-domain environmental information, such as geographical features, available services, spectral regulations, location of various entities of interest, i.e. radios, reflectors, obstacles, plus radio equipment capability profiles, relevant policies and past experiences. The REM information can be updated with observations from $\mathrm{CR}$ users and disseminated throughout $\mathrm{CR}$ networks.

The main contributions of this paper are:

- The Scenario and problem statement definition for the proposed Cognitive RRM. In the considered scenario, there is no direct coordination between PU and CR networks. Therefore, CR users access the spectrum in an opportunistic manner depending on the information provided in the REM, such as geo-localized PU spectral activities, to estimate the available network resources. The objective of the Cognitive RRM is to maximize the spectral resource utilization. This is equivalent to minimize the difference between the total available capacity and the achievable CR data rates while satisfying $\mathrm{CR}$ demands and interference constraints.

- The System architecture of the Cognitive RRM, which is composed of the CR centralized entity and several CR users, with their specific functionalities, i.e. acquiring the measurements from the environment, collecting and storing sensing data, constructing REM, exchanging control information, and coordinating the RRM. After defining the functional blocks, we will present the interfaces between them along with their message exchange to obtain a flexible resource management.

The remainder of the paper is organized as follows: Sec. 2 presents the scenario and problem statement. Sec. 3 describes the proposed system architecture, emphasizing the system requirements and the functionalities of the network elements. Sec. 4 details the interfaces between the functional entities. Sec. 5 describes the protocol and messages sequence chart to realize the proposed Cognitive RRM, and finally Sec. 6 presents the conclusions.

\section{SCENARIO AND PROBLEM STATEMENT}

In this section, the considered scenario of Non-coordinated Spectrum Access between PUs and CR users is described. In this scenario, there is no coordination between PU and $\mathrm{CR}$ networks. Therefore, $\mathrm{CR}$ users access the spectrum in an opportunistic manner depending on the information provided in the REM, such as geo-localized spectral activities, which are used to estimate the available network resources. Here we consider a Cognitive RRM design [6] that takes into account heterogeneous PUs with different spectral features. By exploiting these features, the RRM framework can improve the efficiency of spectrum sharing in CR networks and, thus, enhance their capacity. In this context, the RRM calculates the available capacity of the CR network based on the different existing PUs. After the detection and classification process of heterogeneous PUs, the useful features are provided by the REM, such as the PU bandwidth, the allowed interference and the activity pattern, valid for the geographical area where CR users operations are carried out. These PU features are used to calculate the available capacity for CR users. The RRM control mechanism regulates the sharing of the total available capacity among CR users in accordance with their variable data rate requirements. A cluster of CR users that share the same available resources is formulated around each PU type. In particular, the proposed RRM will identify different PU types with their associated CR clusters by exploiting REM information. Note that clusters are not determined by the location of their CR users but by a specific PU type.

The Cognitive RRM consists in an optimization framework that aims to minimize the difference between the available capacity and the achievable data rate while satisfying CR demands and interference constraints. The RRM is comprised of two stages:

- RRM step 1: RRM assigns CR users to their appropriate clusters based on the demands of CR users and available capacity in the cluster (Admission Control Policy);

- RRM step 2: RRM allocates the required resources, in terms of transmission power, time and bandwidth, for the newly assigned CR users to the cluster based on Orthogonal Frequency Division Multiple Access (OFDMA) technique.

As shown in Fig. 1, different PU types with their associated $\mathrm{CR}$ clusters are identified. The network is composed of CR users and a CR centralized entity, which can be located in a CR base station (BS). Specifically, the CR centralized entity is responsible for collecting sensing data, constructing REM, and coordinating the RRM. It is composed of:

- the REM Manager, which has the data elaboration functionality for processing the measurements to construct the REM,

- the REM Storage and Acquisition (SA) unit, which acquires sensing data from CR users and saves it as a local database. Also the REM, built by the REM manager through the data processing, is stored in the REM SA.

The REM is then used by the CR centralized entity to perform the resource management.

The scenario illustrated in Fig. 1 shows three types of PUs, e.g., WiMax, LTE and IEEE 802.11. These PU types form three different clusters of CR users, which can overlap in space as can be seen in Fig. 1. Specifically, the measurements obtained by CR users are collected in the REM SA and processed by the REM manager to detect and classify the existing PU types in the considered geographical area. The detection and classification process [5] used is not detailed in this paper because it is out of the scope of this work. After the classification process, the REM manager updates 


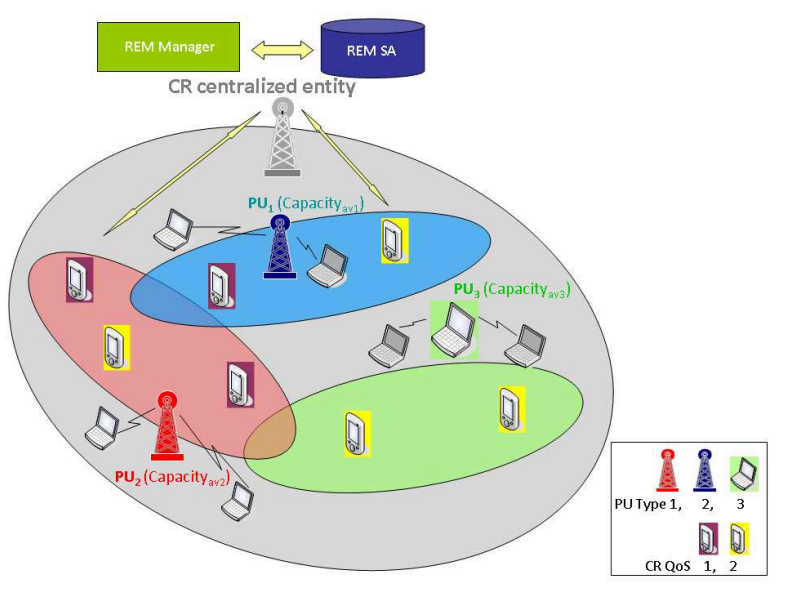

Figure 1: Proposed System Architecture

the REM with the identified PU types and the associated features: bandwidth, allowable interference level and activity pattern. These features are useful for the calculation of the available capacity in a given cluster, as explained in [6]. Also, CR users that share the capacity of a cluster can have different QoS. Fig. 1 considers two types of CR QoS, named CR QoS 1 and CR QoS 2 respectively.

\section{SYSTEM ARCHITECTURE}

As shown in Fig. 1, the proposed CR network is infrastructurebased with a CR centralized entity that coordinates the resource allocation for CR users. Before describing in detail the system architecture, in the following we summarize the useful data stored in the REM SA.

\subsection{Information/data model}

The REM SA stores sensing data plus other information useful for the RRM process. Specifically, the REM SA stores three types of information:

1. Information about CR users, including location information and type of detection. In this context, the cyclostationary feature detector is used because it is able to detect and classify heterogeneous PUs.

2. Raw data measurements, i.e. sensing information sent by CR users to the CR centralized entity. In particular, measurement data depend on the employed detector. The maximum of the autocorrelation function and the interval time in which this maximum is detected [5] are the type of information stored for the considered scenario.

3. REM, built after the data processing of the REM manager. More details on the REM construction will be provided in the following sections. The data stored in the REM are the features of heterogeneous PUs and the propagation features. Specifically, after receiving the sensing information about the detected and classified PUs, the REM manager extracts the PU features, which will be used to efficiently allocate radio resources among CR users. The values of these features are specified in different PU standards, and they are briefly summarized as:
- PU allowed interference levels (to calculate available capacities and to adapt CR transmission power);

- PU activity patterns (to calculate available capacities and to adapt CR transmission time);

- PU bandwidth (to calculate available capacities and to adapt CR bandwidth);

- propagation features, such as the propagation factor, (to calculate CR transmission power).

This information is valid for the geographical area where the operations of CR users are applied. These PU features stored in the REM are used in the Cognitive RRM framework to coordinate the sharing of radio resources among CR users.

\subsection{System Requirements}

In the considered scenario, i.e. non-coordinated spectrum access between PUs and CR users, the entire management process is based on the CR centralized entity. Thus, the CR users have to be located in the transmission range of the CR centralized entity and vice versa for two reasons:

- to send sensing information to CR centralized entity,

- to receive REM information from the CR centralized entity (e.g., CR clustering information and power-frequency allocation information).

Moreover, there is a number of functional requirements at the REM SA and REM manager, which are necessary to manage the complexity of resource allocation. These requirements basically consist in knowing a set of measurements and information needed by the processing and allocation algorithms in RRM (e.g., utilized bandwidth, allowed interference, PU location).

\subsection{Functionalities of the Network Entities}

As shown in Fig. 1, the network is composed of CR users and a CR centralized entity, which require a number of functionalities to carry out the RRM procedure. The following Fig. 2 shows in detail the functionalities of each network entity. In particular, the grey CR base station and the yellow and purple CR users are the CR network elements of Fig. 1 , while the blue boxes represent their functional blocks. Moreover, Fig. 2 shows the interfaces among the different entities, which will be described in the following Sec. 4 .

Going into detail, CR users are the network entities with the sensing functionality, so they represent the measurement capable devices (MCDs) responsible for acquiring the measurement information from the environment. In particular, the CR users utilize a feature detector [5] able to detect and classify heterogeneous PUs. This information about different detected PUs is sent to the CR base station through the air interface (Itf $A$ ). The CR base station is the entity that collects sensing data, constructs REM, and coordinates the RRM. In particular, the BS functionality responsible for collecting the sensing data from CR users is the REM Acquisition unit. This unit also sends the registration information from CR users to the REM manager and the measurement requests from the REM manager to CR users. The interface in charge of communicating between the REM Acquisition 
and the REM manager is the interface REM manager-REM acquisition (Itf $M A$ ). The REM manager is the core functionality of the BS because it is able to build the REM using the sensing data coming from the REM acquisition unit. The REM is thereby sent to the REM storage for the saving process and to the REM RRM for executing the resource allocation. The interface REM manager-REM storage (Itf $M S$ ) is responsible for the exchange of control information, such as read and write messages, and data messages between the REM manager and the REM storage, while the interface REM manager-REM RRM (Itf $M R$ ) is in charge of exchanging control and data information between the REM manager and the REM RRM in order to obtain an efficient management of the radio resources among $\mathrm{CR}$ users.

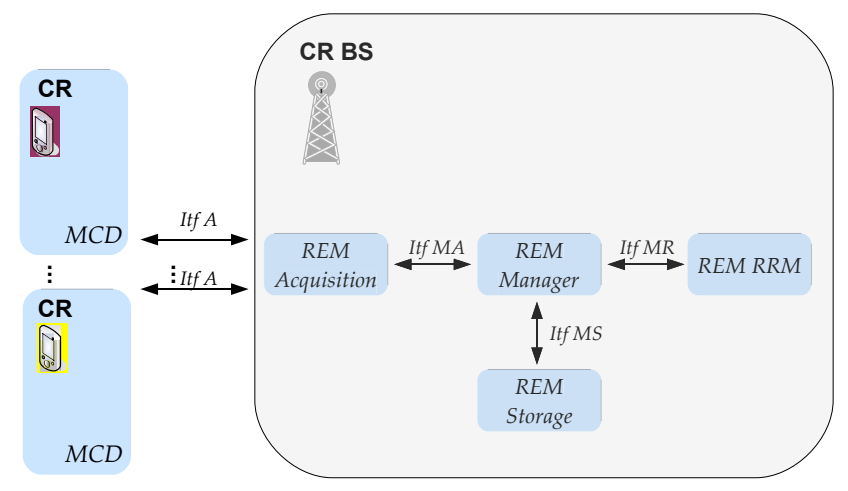

Figure 2: The functionalities of the network entities to carry out the Cognitive RRM procedure

\section{INTERFACES BETWEEN THE FUNCTIONAL ENTITIES}

As shown in Fig. 2, the functional entities communicate among them through specific interfaces. Through these interfaces, several types of messages are exchanged. Fig. 3 depicts a message exchange process between the functional entities to carry out the RRM procedure. These interfaces are described in details in the following.

- The air interface (Itf $\boldsymbol{A}$ ) is the interface between the MCDs, i.e. the CR users, and the REM acquisition unit. As shown in Fig. 3, this interface is used to exchange both control and data messages. The control messages are registration messages and measurement requests, while the data messages are raw measurement data from CR users and measurement report from the REM manager after the data processing, i. e. the built REM.

- The interface REM manager-REM acquisition (Itf $M A$ ) is in charge of sending control and data messages between the REM acquisition and the REM manager. Fig. 3 shows that the control messages on this interface are the registration information that the REM acquisition receives from the CR users and sends to the REM manager, and the measurement requests from the REM manager to the REM acquisition after the REM quality check. The data messages exchange consists in sending the raw data from the REM acquisition to the REM manager for the data processing, and in sending back the built REM, i. e. the measurements report.
- The interface REM manager-REM storage (Itf $\boldsymbol{M S}$ ) is responsible for the exchange of control messages between the REM manager and the REM Storage, e.g. read or write messages, and for the data exchange, e. g. the information of the REM built by the REM manager and sent to the REM storage for the saving process.

- The interface REM manager-REM RRM (Itf $M R$ ) is used to send the measurement results, after the data processing of the REM manager, to the RRM. As shown in Fig. 3, the obtained REM is exploited for the resource allocation among CR users, which consists of two steps: the allocation of CR users to clusters and the allocation of resources to CR users inside the clusters, in terms of transmission power, time and bandwidth. More details on this process are given in $[6]$.

\section{MESSAGE SEQUENCE CHART}

The proposed RRM architecture requires specific interactions among its components. This interaction is defined by a number of message exchanging mechanisms. The REM SA deals with two types of messages: the first ones are exchanged with REM manager through Itf $M S$ and Itf $M A$ interfaces, and the other types of messages are exchanged with CR users through Itf $A$ interface.

The messages exchanged between REM SA and CR user are:

- Registration: This message is sent by the CR user to provide CR user-related information such as data rate requirements.

- Measurement Request: Before every resource allocation cycle, the REM manager polls a set of CR measurements through a measurement request message with specific measurement types such as interference and locations.

- Measurement Response: This message is the CR user response to the measurement request message.

- Configuration: This message is sent by the REM manager to assign the CR user with the allocated resource.

Similarly, the messages exchanged between REM manager and REM SA are:

- Update: This message is provided by the REM SA upon changes occur in their database contents or as a response to some report messages.

- Report: The report message is sent by the REM manager to trigger some processing tasks such as PU activity estimation.

Fig. 3 details the message exchange between different functionalities to carry out the RRM procedure. In Fig. 3, the REM acquisition and the REM storage functionalities are merged together in the REM SA module. Going into detail, the CR users send their registration information to the REM SA. In particular, only the REM acquisition functionality of the REM SA is involved in this step, sending 
the received registration information to the REM manager to update the number of CR users involved. At this step, the REM manager reads the stored information in the REM storage through the Itf $M S$ interface and carries out the REM quality check to evaluate the need of new measurements. Thus, if it is necessary, the REM manager requests new measurements to the REM acquisition through the Itf $M A$ interface, which collects the sensing data from CR users through the Itf $A$ interface. Specifically, the REM acquisition sends the measurements' requests to the CR users first, and then it receives the obtained measurements from them. After that, the REM acquisition forwards these data to the REM manager through the Itf $M A$ interface, which processes the data to build/update the REM. The REM manager extracts the features of heterogeneous PUs from the sensing data, and uses these features to build the REM and to calculate the available capacity for CR users. This information is essential for the RRM process, which is the final goal of the proposed framework. After the processing, obtained data are stored in the REM storage through the Itf $M S$ interface and are sent to the CR users by the REM acquisition through the Itf $A$. At this point, the CR users can adapt their transmission parameters accordingly, as explained in [6], and the RRM procedure is carried out through the Itf $M R$ interface. Fig. 3 shows the two steps of the RRM: the allocation of $\mathrm{CR}$ users to clusters, and resource allocation inside the clusters, in terms of transmission power, time and bandwidth, as detailed in [6].

\section{CONCLUSION}

In this paper, a system architecture for Cognitive RRM has been developed. First, we described the scenario and problem statement in details. In particular, the key point of the RRM approach is the exploitation of multiple features of heterogeneous PUs, stored in a REM, and variable CR rate demands for the efficient utilization of spectrum resources. Then, we defined the requirements of the system, and the functionalities of network entities were fully analyzed. Moreover, we presented the interfaces between the functional blocks and the protocol for the message exchange in order to carry out a flexible and efficient Cognitive RRM.

\section{REFERENCES}

[1] I. F. Akyildiz, W.-Y. Lee, M. C. Vuran, and S. Mohanty "Next generation/dynamic spectrum access/cognitive radio wireless networks: a survey," Computer Networks, Elsevier, Vol. 50, no. 13, pp. 2127-215, Sept. 2006.

[2] Ahmed, M.; Kolar, V.; Petrova, M.; Mahonen, P.; Hailes, S.; , "A component-based architecture for cognitive radio resource management," Cognitive Radio Oriented Wireless Networks and Communications, 2009. CROWNCOM '09. 4th International Conference on , pp.1-6, 22-24 June 2009.

[3] M. Petrova, and P. Mahonen, "Cognitive Resource Manager: a cross-layer architecture for implementing cognitive radio networks," in: F. Fittzek, M. Katz (Eds.), Cognitive Wireless Networks, Springer, 2007.

[4] M. Petrova, P. Mahonen, J. Riihijarvi, M. Wellens, "Cognitive Wireless Networks: Your Network Just Became a Teenager" poster in IEEE INFOCOM, Barcelona, April 2006.

[5] A. Vizziello, I. F. Akyildiz, R. Agusti, L. Favalli, and P. Savazzi, "OFDM Signal Type Recognition and Adaptability Effects in Cognitive Radio Networks," in

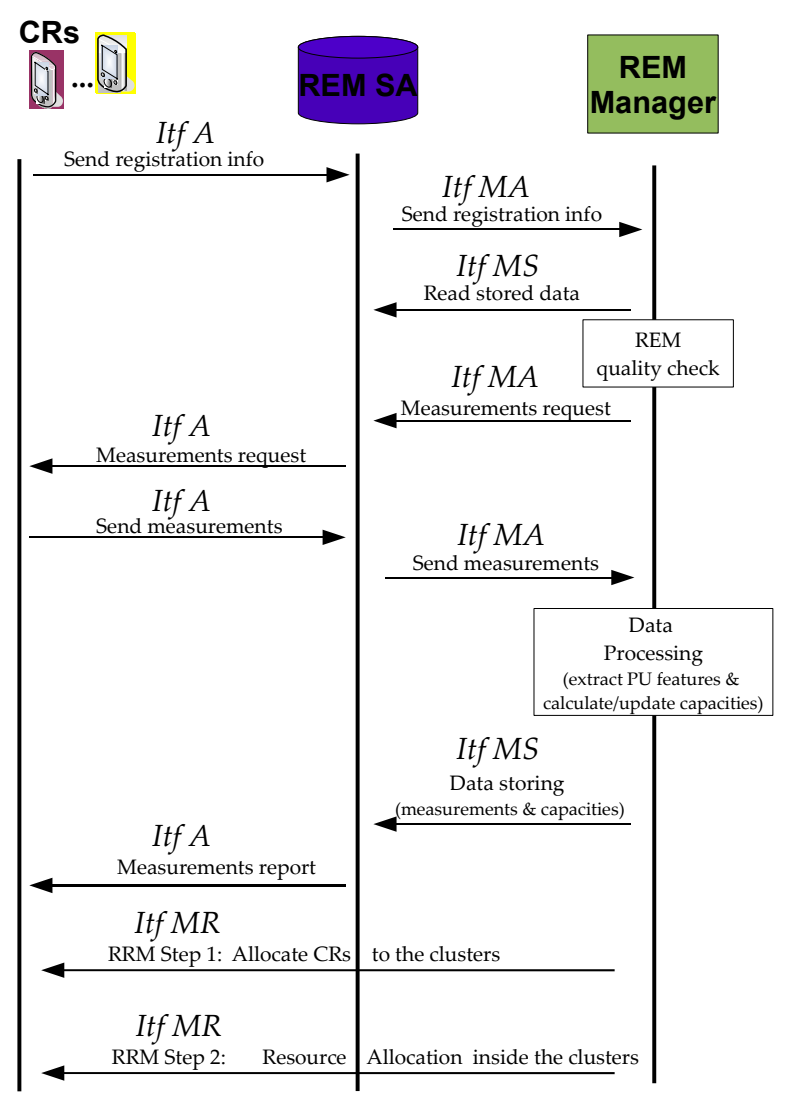

Figure 3: Message Sequence Charts to carry out the Cognitive RRM procedure

Proc. of IEEE GLOBECOM 2010, Miami, Florida, USA, 6-10 Dec. 2010.

[6] A. Vizziello, I. F. Akyildiz, R. Agusti, L. Favalli, and P. Savazzi : "Cognitive Radio Resource Management exploiting Heterogeneous Primary Users," to appear in Proc. of IEEE GLOBECOM 2011, Houston, Texas, USA, 5-9 Dec. 2011.

[7] B. Wang, K. J. R. Liu, "Advances in Cognitive Radio Networks: A Survey," Selected Topics in Signal Processing, IEEE Journal of, vol.5, no.1, pp.5-23, Feb. 2011. 